\title{
ILCEA
}

Revue de l'Institut des langues et cultures

d'Europe, Amérique, Afrique, Asie et Australie

$15 \mid 2012$

Les mots de la crise

\section{Crise financière et langue de spécialité : les mots des maux ou le dire d'une nécessaire vulgarisation}

Specialised English terms related to the financial crisis and the resulting discourse of popularisation in German

\section{Claire Allignol et Elodie Vargas}

\section{OpenEdition}

\section{Journals}

Édition électronique

URL : http://journals.openedition.org/ilcea/1177

DOI : 10.4000/ilcea. 1177

ISSN : 2101-0609

Éditeur

UGA Éditions/Université Grenoble Alpes

Édition imprimée

ISBN : 978-2-84310-220-2

ISSN : 1639-6073

Référence électronique

Claire Allignol et Elodie Vargas, "Crise financière et langue de spécialité : les mots des maux ou le dire d'une nécessaire vulgarisation », ILCEA [En ligne], 15 | 2012, mis en ligne le 30 janvier 2012, consulté le 20 avril 2019. URL : http://journals.openedition.org/ilcea/1177 ; DOI : 10.4000/ilcea.1177

Ce document a été généré automatiquement le 20 avril 2019

(c) ILCEA 


\section{Crise financière et langue de spécialité : les mots des maux ou le dire d'une nécessaire vulgarisation}

Specialised English terms related to the financial crisis and the resulting discourse of popularisation in German

Claire Allignol et Elodie Vargas

\section{NOTE DE L'AUTEUR}

Les auteurs expriment ici leur gratitude envers les deux relecteurs anonymes de ce travail et tiennent à les remercier pour leurs observations et suggestions très constructives.

\section{Introduction}

1 Comment parler des phénomènes de la crise financière, ou plutôt : comment trouver les mots du Dire quand des évènements de l'actualité viennent solliciter un domaine de spécialité?

2 Cette contribution se propose d'analyser - en termes d'outils de transmission de l'information - les discours dans la presse allemande relatant la crise de 2008 lors de ses premiers signes visibles pour la société. C'est ainsi que nous nous intéresserons au cadre d'émergence du discours, à la nature même de ce discours et aux outils de celui-ci, afin de dresser un portrait le plus complet possible du traitement des termes étrangers au lecteur profane. Le corpus à la base de ce travail se compose d'articles issus de quotidiens allemands sur une période allant du $1^{\mathrm{er}}$ janvier 2006 au 31 décembre 2009. Les communiqués des Agences de Presse allemandes, ainsi que la presse spécialisée économique allemande ${ }^{1}$, ont aussi fait l'objet d'analyses sur la même période, dans une optique comparatiste ${ }^{2}$. En effet, l'observation des communiqués de presse allemands, 
anglais et espagnols du domaine économique ${ }^{3}$ ont permis de faire apparaitre certains termes comme récurrents, leur emploi comme massif et ciblé. Leur recherche dans un corpus de journaux spécialisés, puis dans un corpus de quotidiens et hebdomadaires a confirmé leur forte présence sur une période allant de février-mars 2007 à fin 2009. Ainsi, il est apparu comme possible de définir un « mot de la crise » comme étant « un terme issu de la terminologie anglo-américaine, qui connait un pic d'utilisation durant la crise immobilière et financière et qui n'est donc pas nécessairement un terme nouveau ». Les termes retenus sont Hedge Fund, Mortgage, Bad bank, Subprime/ Subprime loan, Bubble, Recession, Toxic assets et Securitization. Cet article ne pourra traiter en détail chaque terme, mais s'appliquera à montrer les fonctionnements communs à tous à l'aide de termes prototypiques.

\section{La crise immobilière et financière : une nécessaire transmission, mais pour quelle vulgarisation?}

3 Les termes subprime, mortgage, etc. ont fait leur apparition dans la presse quotidienne lorsque les premiers signes de la crise financière et immobilière américaine ont été visibles, et que, dans la société mondialisée qui est la nôtre, il a été nécessaire de parler de ces phénomènes et de mettre en mots une certaine technicité financière et immobilière, domaines inconnus du grand public jusque-là, surtout en Europe. La mise en accessibilité à ce type de contenu pour un public profane pose la question de la nature de ces communications et donc le problème de la vulgarisation.

4 La vulgarisation scientifique et technique vise, à tous les niveaux de la population, à freiner, voire à résorber ce que l'on appelle une «aliénation culturelle» et à " acculturer» le public profane $e^{4}$. Une fonction sociale se dégage ainsi, consistant à démocratiser le savoir en mettant le public en contact avec les sciences et techniques qui lui sont obscures. Cette remarque sur la science est applicable à toute catégorie du monde. Un vocabulaire spécifique et précis est nécessaire afin d'identifier et dénommer ce qui existe, ce que l'on découvre ou que l'on invente au fil du temps (quel qu'en soit le domaine). C'est ainsi qu'apparaissent des concepts spécialisés, propres à chaque découverte, invention, etc., et des termes les désignant. Cependant, ces termes et le discours de spécialité les véhiculant s'avérant trop cryptiques pour constituer un outil de communication universel, les termes spécifiques employés sont souvent taxés de 'jargon' par les membres extérieurs à la communauté ${ }^{5}$. C'est justement parce qu'ils peuvent poser des problèmes de compréhension, que ces termes spécialisés constituent, pour une bonne part, la raison d'être de la vulgarisation (outre les autres problèmes liés à l'opacité du discours due à certains présupposés connus des seuls spécialistes). La vulgarisation se propose ainsi, par une mise en contact du public profane avec le savoir spécialisé, de faire progresser la connaissance (et la culture) des membres qui le constituent, un contact direct semblant impossible. En effet, l'incompréhension des termes spécialisés provoque au moins deux blocages : premièrement, la communication entre les différents acteurs de la société est tout d'abord entravée du fait du caractère étranger des termes employés ; en reprenant les mots de J. Authier-Revuz (1982: 34) sur la vulgarisation scientifique, mais en élargissant à d'autres domaines de spécialité , on peut en effet dire que « la 'langue' [...] devenant hors des murs de la communauté une langue étrangère, une rupture se produit dans l'intercompréhension ». Deuxièmement, le rapport à certaines connaissances pour les non-spécialistes - c'est-à-dire la grande majorité de la population -, est compliqué par 
une attitude de rejet du discours spécialisé. En effet, comme le rappelle très justement M.F. Mortureux, "une opinion très répandue voit précisément dans l'existence des terminologies, désignées alors du terme péjoratif de "jargons", un obstacle majeur, sinon l'obstacle principal, qui interdirait à tout un chacun l'accès direct aux discours primaires » $(1988: 120)$.

5 Mais comment situer les discours sur la crise par rapport à la vulgarisation? Dans son aspect terminologique, par l'offre d'un discours rapporté, d'un discours traduit, d'une véritable reformulation du discours spécialisé dans laquelle il s'agit d'établir un « contact entre terminologie et lexique général » (Mortureux, 1988:123), on peut dire que les diverses communications journalistiques sur la crise immobilière et financière relèvent de la vulgarisation. Des éléments factuels permettent, cependant, de limiter le champ et d'apporter quelques restrictions.

6 Comme le fait remarquer Jurdant (2009), la vulgarisation, à l'époque de son apparition, ne résulte pas d'une demande. En effet, lorsque la vulgarisation naît à la fin du Moyen Âge en Europe, et s'affirme avec l'émancipation de la bourgeoisie (particulièrement aux XVII et $\mathrm{xVIII} \mathrm{e}^{\mathrm{e}}$ siècles), elle reste très élitiste, doit être vue comme une ouverture de la science au monde ${ }^{6}$ et surtout, ne répond pas à une demande sociale du peuple. C'est en cela que réside la grande différence avec les discours journalistiques sur la crise, dans la mesure où ceux-ci sont nés, pour leur part, d'un besoin de connaissance et de compréhension. Il faut donc les considérer comme autant de réponses à une demande urgente émanant du public profane.

7 Par ailleurs, s'il est établi que la vulgarisation a une fonction sociale et vise une acculturation du public profane ${ }^{7}$, il semble difficile de parler dans le cas des discours nous occupant, de "démocratisation du savoir » et "d'acculturation ", dans la mesure où les journalistes-vulgarisateurs, en produisant des articles sur la crise, ne se présentent pas "comme les artisans d'une possible révolution du savoir qui vise un partage plus équitable entre tous les acteurs sociaux » (Schiele et Jacobi, 1988:24)8. Si une fonction sociale se dégage pourtant dans ces transmissions journalistiques, il semble plus intéressant de la voir du côté de la vulgarisation telle que la définit Jurdant (2009), à savoir, comme une oralisation. En effet, la vulgarisation doit s'inscrire dans un monde de sens. Or, ce dernier ne peut se constituer que dans le rapport d'un Homme à un autre Homme et par le caractère collectif de sa production. La vulgarisation doit ainsi tendre vers une réflexivité et donc parvenir à une certaine forme d'oralité, afin de devenir un enjeu de la communication des acteurs sociaux entre eux. Au-delà d'une présentation et d'explications de termes et de phénomènes relevant d'un domaine de spécialité, expliquer et parler de la crise ne vise donc pas à participer à la formation culturelle de tout humain dans notre société (contrairement à la conception de la vulgarisation de Schiele et Jacobi, 1988 : 17), mais à produire un discours voulant devenir, par les échanges des profanes, objet de discours et prenant du sens par ceux-ci. C'est ainsi qu'il y a tout d'abord construction d'un discours sur la crise, puis construction d'un savoir sur la crise, formées des étapes suivantes :

- Discours d'expert ;

- Circulation des discours ;

- Stratégies de mises en discours médiatique ;

- Déconstruction du discours médiatique par le citoyen en vue de la construction d'un savoir citoyen. 

demande [...] pour remplir un 'devoir', une 'mission', il se donne [...] un statut ambigu de commentator-compilator, s'évertuant, dans l'effacement, à mettre les deux pôles en contact » (Authier, $1982: 39$ ).

La dernière distinction qu'il convient de faire avec la vulgarisation scientifique, technique ou la médiation culturelle est que, si celles-ci constituent un outil utile, nécessaire, voire indispensable pour le chercheur, le concepteur ou l'artiste tenant à populariser ses recherches et à se faire ainsi connaître, il est quasiment certain que le monde de la finance - spécialiste du domaine - ne tenait pas à divulguer ses données et ses modes de fonctionnement, bref à vulgariser au moment de la crise. Dans ce cadre-là, il convient de se demander comment se présente ce discours.

\section{Formes discursives}

\section{Reformulations intratextuelles}

Les textes retenus pour cette étude sont ce que l'on nomme en journalistique des synthèses, des filets, des interviews et des semi-interviews ; en allemand: "Berichte ", « Nachrichten » ou « Meldungen » et «Interviews» ${ }^{9}$. La présentation des termes du domaine financier dans ces discours sur la crise financière et immobilière se fait sous différentes formes, dont la plus fréquente est la reformulation intratextuelle.

11 Nous $^{10}$ avons défini la reformulation intratextuelle comme une opération stratégique métalangagière, par laquelle deux segments sont mis en relation le temps d'un discours, le second - renvoyant au même référent - devant être compris comme une formulation autre du premier. L'opération de reformulation, tournée vers un interlocuteur, fondamentalement dialogique, résulte d'un retour réflexif du sujet sur son propre dire et s'accomplit dans l'esprit d'un changement de perspective énonciative. Le second segment - dit segment reformulateur - apportant un supplément d'informations, ajouté mais non obligatoire, est suppressible au niveau phrastique. Le lien qui unit les deux segments est un lien paraphrastique, défini non par rapport à une parenté sémantique, mais par rapport à un apport informationnel ${ }^{11}$.

C'est ainsi que dans l'exemple (1), «hochverzinslichen zweitklassigen Hypothekendarlehen » représente le segment source (en italique), alors que Subprime Loans (en gras) ${ }^{12}$ représente le segment reformulateur. Le marqueur de reformulation est ici typographique : il s'agit des parenthèses.

Allerdings hält die Immobilienkrise in den Vereinigten Staaten an. Nach den Zahlen des Handelsministeriums sind die Wohnausgaben im vergangenen Quartal um 15,8 Prozent gesunken. Kritisch ist die Lage vor allem für Hausbesitzer, die ihr Eigentum mit hochverzinslichen zweitklassigen Hypothekendarlehen („Subprime Loans“) finanziert hatten. (Süddeutsche Zeitung, 29.06.2007)

Dans les exemples de notre corpus, le segment reformulateur véhicule des informations n'entrant pas en contradiction avec celles proposées dans le segment source. Son apport n'est que bénéfique et enrichissant. Les deux segments se situent à un même niveau hiérarchique et le lien les unissant repose alors sur une parenté sémantique réelle, il s'agit donc de reformulations dite paraphrastiques ${ }^{13}$. 

monde décrit ou défini en code courant sous forme de groupes nominaux ou de groupes verbaux le terme anglais spécialisé du domaine le désignant ${ }^{15}$. Ici, le segment reformulateur contenant le terme, le "mot de la crise" subprime mortgages propose une dénomination pour «Hypothekendarlehen niedriger Kreditqualität», contenu dans le segment source. alors introduite par le marqueur lexical « das sind» (ou « das ist / X ist»), comme en témoigne l'exemple (3) ci-dessous :

Damit hat die Hypothekenkrise in den Vereinigten Staaten eine neue Wendung genommen. New Century, ein Finanzdienstleister aus Irvine in der südkalifornischen Boomregion Orange County, ist eine der ersten Adressen im Geschäft mit so genannten Subprime loans ${ }^{16}$, das sind Hypothekendarlehen, bei denen die Bank die Bonität des Kunden nicht genau prüft, dafür aber höhere Zinsen verlangt. Nach Angaben des Branchendienstes Inside Mortgage Finance wurden im vergangenen Jahr 600 Milliarden Dollar an derartigen niederrangigen Immobilien-Darlehen ausgegeben - das ist nicht weniger als ein Fünftel des gesamten Marktes. In normalen Jahren liegt der Anteil bei höchstens fünf Prozent. ( Süddeutsche Zeitung, 06.03.2007)

19

Il n'est pas étonnant de trouver un foisonnement de reformulations concernant les subprime-mortgages, puisque cette modalité de crédit est absente du paysage allemand (et européen) d'une part, et se trouve être une des bases de la crise financière, d'autre part. C'est ainsi que correspondent à subprime loans ou subprime-mortgages « Schlecht besicherte Hypothekenanleihen», "schlecht besicherte Kredite ", «zweitklassige Hypothekenkredite ", "so genannte Sub-Prime-Kredite an Kunden mit schwacher oder sehr schwacher Bonität », « Hypothekengeschäfte minderer Qualität ». 
20 Cette récurrence de périphrases nominales identiques de journaux en journaux ne peut échapper au chercheur lors de l'examen du corpus d'articles journalistiques. C'est la raison pour laquelle nous avons procédé à une analyse systématique des communiqués de presse et des articles paraissant dans les journaux spécialisés d'économie pour la même période définie dans ce travail.

21 Cette recherche nous a permis de constater que les communiqués de presse contiennent déjà toutes les reformulations paraphrastiques en dénomination soulignées ci-dessus, ainsi qu'en témoignent les exemples ci-dessous :

Die Spannungen zwischen den USA und Iran dürften den US- Anleihemarkt am Montag mit Kursgewinnen eröffnen lassen. Londoner Händler verweisen zudem auf wachsende Bedenken, was den US-Markt für zweitklassige Hypothekenkredite ( Subprime Mortgages) angeht. (AWP International, 26.02.2007)

New Century gehört zu den größten Unternehmen auf dem Markt für zweitklassige Hypothekendarlehen („subprime mortgages“) - das sind Kredite, die an Kreditnehmer mit schlechter Bonität ausgegeben werden. (Reuters, 08.03. 2007) New Century gehört zu den größten Unternehmen auf dem Markt für so genannte ” subprime mortgages"oder zweitklassige Hypothekendarlehen und leiht Kreditnehmern mit schlechter Bonität Geld. (Reuters, 10.03.2007)

Zentrales Thema war die Krise der sogenannten Subprime LendersImmobilienfinanzierer, die Darlehen an Schuldner von geringerer Bonität vergeben. (Reuters, 12.03.2007)

Allerdings sind diese zur Zeit in den USA stark unter Druck: Einige Institute, die zweitklassige Hypothekendarlehen- so genannte subprime mortgages - anbieten, stehen kurz vor der Pleite. Das sind Darlehen, die an Kreditnehmer mit schlechter Bonität ausgegeben werden ${ }^{17}$. (Reuters, 14.03.2007)

L'observation de la presse spécialisée souligne la même tendance, comme le montre l'exemple (9) :

Wie es gestern hieß, untersucht die Staatsanwaltschaft den Fall des US - Finanzierers New Century Financial- die Nummer zwei im Geschäft mit Immobilienkrediten an Kunden mit geringer Bonität(„Subprime-lending“). Financial Times Deutschland, 06.03.2007)

S'il est évident, pour qui connaît le fonctionnement de la presse, que les brèves, les filets, les synthèses, etc. («Meldungen » / " Nachrichten », Berichte », etc.) ont pour base les divers communiqués de presse ${ }^{18}$, ces exemples montrent bien que les vrais journalistesvulgarisateurs ne sont pas ceux des différents journaux, mais bien ceux travaillant en amont au sein de l'Agence de presse. La reformulation est donc une mise en discours qui se fait bien en amont et qui est simplement reprise ensuite dans les différentes rédactions.

24 À ce propos, il est intéressant de noter que les termes anglais Bubble et Recession n'apparaissent pas dans les quotidiens allemands sous cette forme, mais qu'ils ont été traduits et employés tels quels, c'est-à-dire sans reformulation intratextuelle et interlinguale. Les termes en allemand sont respectivement: "Blase » et "Rezession». Notons que la traduction «Verbriefung » pour Securitization n'a jamais été très employée, la traduction atteint un pic fin 2007 et connaît ensuite une décroissance assez rapide. Le terme Toxic assets ne fait pas non plus l'objet de reformulation intratextuelle, il est utilisé directement en allemand sous la forme «toxische Wertpapiere" ou «faule Wertpapiere ». Dans le cadre de nos observations concernant les communiqués de presse, ceci est particulièrement intéressant : en effet, les adjectifs «faul » ou " toxisch » sont connotés négativement et expriment un jugement de valeur. Or, une agence de presse se 
doit d'être neutre ${ }^{19}$ et il est donc impossible que ces termes se trouvent dans ses communiqués, sous quelque forme que ce soit. Cela explique qu'ils ne soient pas traduits de l'anglais à l'allemand, ni reformulés dans les articles journalistiques de presse quotidienne, mais juste "présents", majoritairement signalés par des guillemets. D'où l'on peut en conclure que si le modèle n'est pas donné avant, le journaliste de presse quotidienne ne se risque pas à inventer des correspondances répondantes.

Au-delà des reformulations, il est important de noter que les «mots de la crise» (reformulés ou non) sont souvent précédés de l'adjectif "sogenannt-» dans les communiqués de presse, comme dans la presse spécialisée et donc également dans les articles des quotidiens, ainsi qu'en témoignent les exemples (10), (11) et (12) :

Zentrales Thema sind die sogenannten Subprime Lenders - Hypothekenfinanzierer, die sich auf Kunden mit geringerer Bonität spezialisiert haben. (Dow Jones Unternehmen Deutschland, 12.03.2007)

Wie lax die Kreditvergabe während des US-Immo-Booms war, zeigt auch das Beispiel sogenannter „Piggyback“-Darlehen. (Wirtschaftsblatt, 09.03.2007)

Das Grundproblem ist, dass vor allem in den USA viele Kredite an Menschen vergeben werden, die sie voraussichtlich nie zurückzahlen können. Der dahinterstehende sogenannte subprime lending sector hat im Immobiliensektor inzwischen einen Anteil von 22 Prozent erreicht. Die Leute können ihre Darlehen nur bedienen, wenn die Häuserpreise steigen oder zumindest nicht fallen. Sie sinken jedoch, der Kreditausfall ist damit programmiert. (Süddeutsche Zeitung, 01.03.2007)

«Sogenannt-» ne sert pas ici à signifier au lecteur que l'appellation ne convient pas, parce que mal adaptée à la réalité de l'objet du monde auquel elle s'applique. Il marque au contraire que le terme convient parfaitement, en précisant - et c'est là sa caractéristique principale -, que ce nom-là est conféré par une instance extérieure habilitée à dénommer. Il souligne le terme spécialisé et, ce faisant, s'en distancie en même temps en montrant la source de l'appellation. Le journaliste-vulgarisateur, bien qu'opérateur, se distancie, s'efface devant ce que l'on peut appeler «l'autorité dénommante ${ }^{20}$ » et devient écrivainrelais. Il n'est donc plus qu'un locuteur retranscrivant les paroles d'un énonciateur ${ }^{21}$.

Dans cette chaîne de diffusion de l'information, on peut constater que le terme subprime couplé à « Krise » apparaît au début juillet 2007 dans les communiqués des agences de presse. Il est y très rapidement utilisé sous forme de composé et n'est alors plus reformulé. Environ deux mois plus tard, le même phénomène se reproduit dans la presse spécialisée et il faut attendre fin 2007 pour que le terme subprime ne soit plus reformulé dans les quotidiens et apparaisse couplé à "Krise». Cela est intéressant, car assez révélateur d'un mode de fonctionnement. En effet, le discours de vulgarisation, selon de nombreux auteurs ayant étudié la vulgarisation (voir supra), se marque par le fait qu'il vise une certaine intégration: après avoir expliqué, précisé, le discours intègre ce qu'il vient de désigner comme étranger et le terme marqué est repris sans reformulation, passant ainsi à l'intérieur du discours et n'étant plus stigmatisé comme "venant d'un Autre". Le texte passe donc à un autre niveau en permettant, par un effacement progressif des suites, l'appropriation des termes par le lecteur. Les reformulations ayant accompli la tâche qui leur était impartie, elles peuvent s'effacer au fil du texte. Si ces affirmations sont fausses pour les ouvrages de vulgarisation et certaines émissions de vulgarisation $^{22}$, l'étude de nos exemples corrobore ces affirmations pour ce qui est des articles de presse ${ }^{23}$ sur une période que l'on peut compter en mois. On constate même que cette intégration peut se faire tellement naturellement que certains composés se font sur 
une base de termes anglais-allemands dans la suite du texte, comme en témoigne le terme « Suprime-Darlehen » dans l'exemple (13):

S\&P kündigte am Dienstag an, Anleihen mit einem Nennwert von zwölf Milliarden Dollar auf eine Herabstufung hin überprüfen zu wollen. Dabei handelt es sich um Schuldverschreibungen, die mit Hypothekendarlehen niedriger Kreditqualität (,subprime mortgages“) besichert sind. Grund für die Überprüfung, die mit einer Änderung der Rating-Methodik einhergeht, sei die Erwartung, dass es bei den Subprime-Darlehen $\mathrm{zu}$ wachsenden Ausfällen kommen werde. (Süddeutsche Zeitung, 11.07.2007)

S'il est acquis que toute reformulation est synonyme d'altérité sémantique, notons toutefois que certaines reformulations intratextuelles sont parfois fautives, comme dans l'exemple ci-dessous extrait de la presse spécialisée :

Ein Grund für die Eintrübung des Häusermarkts sind die höheren Hypothekenzinsen. Laut Volkswirt Hooper dämpfen diese einerseits die Nachfrage nach Häusern. Andererseits führten sie verstärkt dazu, dass vor allem Schuldner mit niedrigerer Bonität (Subprime) ihre Kredite nicht mehr bedienen können und die Zahl der Zwangsvollstreckungen zunimmt - was das Angebot erhöht. (Financial Times Deutschland, 19.07.2007)

Il n'y a pas ici identité référentielle, puisque le soi-disant segment reformulateur subprime désigne un type de crédit, alors que le segment source "Schuldner mit niedrigerer Bonität » désigne un référent animé, humain, bénéficiaire dudit crédit. Un raccourci abusif est donc fait, qui amène le profane à une mauvaise interprétation du « mot de la crise ». Comment expliquer un tel phénomène? Cette correspondance malheureuse s'inscrit dans le procédé décrit précédemment, selon lequel le journaliste se sert des communiqués de presse pour en faire des copiés-collés. En effet, on trouve dans le communiqué émanant de Reuters la veille la même reformulation :

Die US-Wirtschaft wird nach Ansicht von Notenbank-Chef Ben Bernanke durch den rasanten Anstieg von säumigen Schuldnern im Zuge der US-Hypothekenkrise nicht ernsthaft in Mitleidenschaft gezogen. Die Auswirkungen der Krise am Markt für Kreditnehmer mit niedriger Bonität (Subprime Mortgage) werde auf den USImmobiliensektor beschränkt bleiben, sagte Bernanke am Donnerstag auf einer Konferenz der Notenbank von Chicago. (Reuters, 18.07.2007)

La reformulation intratextuelle n'est pas le seul moyen d'entourer et de présenter les «mots de la crise ». Les anaphores nominales, parfois en chaîne, sont également un moyen de diffusion des termes.

\section{Chaînes anaphoriques}

Damit hat die Hypothekenkrise in den Vereinigten Staaten eine neue Wendung genommen. New Century, ein Finanzdienstleister aus Irvine in der südkalifornischen Boomregion Orange County, ist eine der ersten Adressen im Geschäft mit so genannten Subprime loans, das sind Hypothekendarlehen, bei denen die Bank die Bonität des Kunden nicht genau prüft, dafür aber höhere Zinsen verlangt. Nach Angaben des Branchendienstes Inside Mortgage Finance wurden im vergangenen Jahr 600 Milliarden Dollar an derartigen niederrangigen ImmobilienDarlehen ausgegeben - das ist nicht weniger als ein Fünftel des gesamten Marktes. In normalen Jahren liegt der Anteil bei höchstens fünf Prozent. Die riskanten Kredite bescherten den Anbietern zunächst enorme Gewinne. Viele senkten die Standards für die Bonität ihrer Kunden in den ersten Monaten des vergangenen Jahres weiter, um neue Kunden zu gewinnen. (Süddeutsche Zeitung, 06.03.2007) 
31 L'exemple (16) ci-dessus montre que le groupe nominal ${ }^{24}$ «so genannten Subprime loans» est repris une première fois par le groupe nominal « Hypothekendarlehen, bei denen die Bank die Bonität des Kunden nicht genau prüft, dafür aber höhere Zinsen verlangt». Nos analyses précédentes ont montré qu'il s'agissait d'un segment reformulateur. Celui-ci est anaphorique dans un rapport d'anaphore infidèle, mais de représentation totale ${ }^{25}$. On constate cependant qu'il n'est pas le seul, puisque « derartigen niederrangigen Immobilien-Darlehen » et « die riskanten Kredite » sont également des anaphores de "so genannten Subprime loans". Bien que non reformulateurs ${ }^{26}$, ces groupes ont tous une fonction de reprise. Il y a coréférence, chaque groupe nominal désignant le même référent, même si les anaphores sont infidèles. En reprenant les termes de Jacobi, on peut dire que subprime loans fonctionne ici comme "terme pivot", autour duquel s'articulent les autres groupes. C'est le caractère infidèle de ces unités qui permet un enrichissement sémantique amenant à des contours plus nets du référent. Par l'établissement des paradigmes désignationnels à partir de groupes nominaux apparaissant au fil d'un texte, le lecteur profane peut avoir une image plus complète de ce que désigne tel ou tel « mot de la crise ». Il est important de souligner que ces anaphores permettent des jugements de valeur de la part du rédacteur, un positionnement et une orientation du lecteur (voir l'exemple ci-dessus) ${ }^{27}$. C'est précisément en cela que l'article de quotidien se distingue du communiqué de presse auquel il emprunte pourtant par ailleurs.

Par ces listes de paradigmes désignationnels, mais également par le cotexte et l'environnement sémantique du terme, naissent des vrais champs lexicaux qui rendent possible toute une palette de métaphores.

\section{Champ sémantiques et métaphores}

L'analyse de notre corpus a montré que le terme hedge funds n'est pratiquement pas expliqué dans la presse, comme si tout un chacun savait exactement de quoi il s'agissait. Cependant, on constate, si l'on s'intéresse à son environnement immédiat dans les textes, qu'il est associé à des substantifs comme "Turbulenzen», "Pleite», «Kollaps», «Zusammenbruch », « Totalverlust », « Risiken », « Krise », « Blackbox », « Schieflage », des adjectifs (en fonction adjectivale ou adverbiale) tels que « angeschlagen», «kaum durchschaubar ", "schlingernd», « hoch spekulativ», "umstritten ». Le contexte est donc souvent négatif, pouvant aller jusqu'à comparer les hedge funds à des sauterelles qui s'abattent sur les entreprises pour les racheter et les revendre dans le seul but de réaliser un profit à court terme. À noter encore, le composé "Geier-Fonds", ou «fonds de vautours ", qui exprime avec plus de force encore la voracité et l'égoïsme de ces charognards.

Dans le même esprit, bad bank est un exemple intéressant. En effet, lorsque la Chancelière Angela Merkel a exposé son projet de créer des sociétés de défaisance pour sortir les actifs toxiques des bilans des banques, elle a employé un terme spécifique "Zweckgesellschaft », neutre et assez peu évocateur. Or, ce terme n'a eu aucun succès (on n'en relève que peu d'occurrences), alors que le terme bad bank -qui, de par son sémantisme, a permis des comparaisons avec des décharges pour déchets toxiques -, s'est maintenant imposé, puisqu'on parle même de la loi sur les bad banks («BadbankGesetz »). 

critique acerbe du système financier, devenu l'ennemi. Ainsi les champs sémantiques de la maladie, des ruines (avec réminiscences historiques), des déchets et de la pollution de l'environnement, des jeux de hasard et des prédateurs (requins, vautours) sont convoqués.

C'est ce que montre l'exemple (17) relevant du domaine médical :

Die Weltwirtschaft ist von einer Seuche befallen. Nach langer Inkubationszeit, Investmentbanken und Versicherer waren bereits infiziert, nahm die weltweite Epidemie mit dem Zusammenbruch von Lehman Brothers ihren Anfang. Jetzt rast die Todeswelle um den Globus. Banken, Autohersteller, Computerfirmen - das Rückgrat der Weltwirtschaft ist in Auflösung begriffen. Angesichts der dem Tode nahen Intensivpatienten, die fast täglich neue Milliardeninfusionen fordern, sehen die geplagten Politiker das Budget für Forschung und Entwicklung (F\&E) meist als Luxus an. (Der Tagesspiegel, 11.03.2009)

Ici, la crise mondiale est comparée à une maladie contagieuse et grave. Le terme "Seuche " s'emploie pour parler des grandes épidémies. Il ne s'agit donc pas d'une épidémie de grippe ni même de rougeole, mais d'une maladie qui a de quoi inquiéter. On parle de morts nombreuses («Todeswelle»), de patients en soins intensifs. Par ailleurs, il est question de patients à l'agonie, de perfusions (de milliards), de période d'incubation et de piqûres financières. journalistes peuvent associer crise et pollution, comme en témoigne l'exemple (18)

Droht bereits die nächste Krise, weil die Finanzbranche weitermacht wie bisher? Besuch in der Deponie: Noch immer ist das Problem der toxischen Wertpapiere inden Bankbilanzen ungelöst. Der Müll lässt sich nur schwer entsorgen. Und neuer Abfall kommt hinzu. Wird der Sondermüll neu verpackt? (Süddeutsche Zeitung, 23.09. 2009)

La crise peut également évoquer un champ de ruines, des substantifs comme "Trümmer ", "Trümmerhaufen », "Trümmer wegfegen / wegräumen» sont alors utilisés, comme autant de réminiscences qui ramènent la crise à la défaite de 1945. Elle peut également emprunter aux jeux de hasard, et des substantifs ou expressions tels « Kasino-Kapitalismus », « Black-Out im Kasino », « Kasino-Mentalität », « das Kapital ins Kasino tragen ", "es geht wieder los, das Kasino ist wieder offen », « die Banken haben sich verzockt », « spekulativen Zockereien » apparaissent alors.

Malgré l'apparente ingéniosité des expressions choisies et des champs sémantiques convoqués, force est de constater que les journalistes-vulgarisateurs n'inventent rien. Les métaphores et les champs lexicaux sont déjà dans les discours des experts, des spécialistes du domaine. C'est ainsi que les métaphores viennent, par exemple, du domaine maritime pour les anglo-saxons ${ }^{28}$. Par ailleurs, l'économie étant assimilée à un organisme, il est normal de trouver des métaphores de la maladie pour en parler.

\section{Conclusion}

41 Cette étude a montré que les «mots de la crise» sont diffusés dans un esprit d'information du lecteur profane, consommateur concerné et demandeur d'information. Mis en exergue comme les " Mots de l'Autre », ils sont présentés massivement sous forme de reformulations intratextuelles paraphrastiques, essentiellement en dénomination, 
mais également en définition. Traduits vers l'allemand ou conservés sous leur forme anglaise, ils sont par ailleurs les mots pivots autour desquels des chaînes d'anaphores s'organisent, donnant ainsi naissance à des champs lexicaux précis et des métaphores ciblées, souvent empreintes d'anthropomorphisme, qui doivent dire la douleur des maux que la crise et ses mots provoquent.

\section{BIBLIOGRAPHIE}

ADAM J.-M., Éléments de linguistique textuelle, Liège, P. Mardaga éd., 1990.

ANDERSON P., ChAUVIn-Vileno A., MAdini M.(éds), Répétition, altération, reformulation, colloque international tenu à Besançon, 22-24 juin 1998, Besançon, Presses universitaires FrancComtoises, 2000.

AUthIER J., « La mise en scène de la communication dans des discours de vulgarisation scientifique », Langue Française, $n^{\circ}$ 53, 1982, p. 34-47.

AuthIER-ReVuz J., Ces mots qui ne vont pas de soi. Boucles réflexives et non-coïncidences du dire, 2 tomes, Paris, Larousse, coll. « Sciences du Langage », 1995.

AuthIER-ReVuz J., « Deux mots pour une chose : trajets de non-coïncidence », dans P. Anderson, A. Chauvin-Vileno et M. Madini (éds), Répétition, altération, reformulation, 2000, p. 37-62.

AuthieR-Revuz J., " Du Dire "en plus" : dédoublement réflexif et ajout sur la chaîne ", dans J. Authier-Revuz et M.-C. Lala (éds), Figures d'ajout. Phrase, texte, écriture, 2002, p. 147-167.

CAUNE J., Pour une éthique de la médiation, le sens des pratiques culturelles, Grenoble, Presses universitaires de Grenoble, 1999.

CAUNE J., « La médiation culturelle : une construction du lien social », Revue les Enjeux de la communication, 2000.

CAUNE J., La démocratisation culturelle : une médiation à bout de souffle, Presses universitaires de Grenoble, coll. « Art, culture, public », 2006.

CHAROLLES M., L'anaphore : problèmes de définition et de classification, Verbum, 1991.

CHAROLLES M., La référence et les expressions référentielles en français,Paris, Ophrys, 2002.

CIAPUSCIO G. E., Wissenschaft für Laien : Untersuchungen zu populärwissenschaftlichen Nachrichten aus Argentinien,Bonn, Romanistischer Verlag, 1993.

Ducrot O. et al., Les mots du discours, Paris, Éditions de Minuit, 1980.

Ducrot O., Le dire et le dit, Paris, Éditions de Minuit, 1984.

FASEL C., Textsorten (Wegweiser Journalismus Band 2), Konstanz, UVK Verlagsgesellschaft, 2008.

JACOBI D., Diffusion et vulgarisation : itinéraire du texte scientifique, Paris, Belles-Lettres, 1986.

JACOBI D., « Le discours de vulgarisation : problèmes sémiotiques et textuels », dans D. Jacobi et B. Schiele (éds), Vulgariser la science : le procès de l'ignorance, 1988, p. 87-117. 
JACOBI D., « "L'homme”. Une nouvelle à caractère scientifique dans la presse et les magazines ", dans S. Moirand, A. A. Bouacha, J.-C. Beacco et A. Collinot (éds), Parcours linguistiques de discours spécialisés, 1994, p. 11-22.

JACOBI D. et SCHIELE B. (éds), Vulgariser la science : le procès de l'ignorance, Seyssel, éd. Champ Vallon, coll. « Milieux », 1988.

JURDANT B., Les problèmes théoriques de la vulgarisation scientifique, Paris, Archives contemporaines, 2009.

KLEIBER G., La sémantique du prototype. Catégories et sens lexical, Paris, Presses universitaires de France, 1990.

KLEIBER G., L'anaphore associative, Paris, Presses universitaires de France, 2001.

LAMIZET B., La médiation culturelle, L'Harmattan, 2000.

MALDIDIER P. et BoltANSKI L., La vulgarisation scientifique et ses agents, Paris, CSE, 1969.

MALDIDIER P. et BOLTANSKI L., La vulgarisation scientifique et son public, une enquête sur Science et Vie, 2 tomes, Paris, CSE-EHESS, 1977.

MAST C. (éd.), ABC des Journalismus - Ein Handbuch (Praktischer Journalismus Band 1), 10., völlig neue Auflage, Bonn, DJV Verlags - und Service GmbH, 2004.

Moirand S., Bouacha A. A., Beacco J.-C. et Collinot A. (éds), Parcours linguistiques de discours spécialisés, Berne, Peter Lang, 1994.

Moles A. A. et Oulif J.-M., « Le troisième homme, vulgarisation scientifique et radio ", Diogène, $\mathrm{n}^{\circ}$ 58, 1967, p. 29-40.

MORTUREUX M.-F., « La vulgarisation scientifique : parole médiane ou dédoublée ? », dans D. Jacobi et B. Schiele (éds), Vulgariser la science : le procès de l'ignorance, 1988, p. 118-148.

NIEDERHAUSER J., Wissenschaftssprache und populärwissenschaftliche Vermittlung. Tübingen, Gunter Narr Verlag, 1999.

VARGAS E., Procédés de reformulation intratextuelle dans les ouvrages de vulgarisation en allemand. Étude d'une opération métalangagière et de ses marques, doctorat d'université de linguistique allemande sous la direction de Madame le Professeur Martine Dalmas, Paris IV Sorbonne, 2005, 1369 p.

VARGAS E., « Les discours de vulgarisation à travers différents médias ou des tribulations des termes scientifiques : le cas de la médecine " [en ligne], ILCEA, 11, Langues \& cultures de spécialité à l'épreuve des médias, sous la dir. de Shaeda Isani, 2009. Disponible sur <http://ilcea.revues.org/ index228.html>

\section{NOTES}

1. Les articles du Financial Times Deutschland n'ont été retenus que lorsque les auteurs étaient allemands afin qu'il ne s'agisse pas de textes traduits. C'est pour cette raison que des articles de Christine Mai-Duc ont été exclus.

2. Dans aucun cas, il n'a été utilisé de traductions, les textes utilisés sont des textes rédigés en allemand.

3. Cette étude repose sur un travail plurilangue, voir les articles dans cette même publication.

4. Sur la vulgarisation, voir E. Vargas (2005), D. Jacobi (1994), D. Jacobi et B. Schiele (1988), J. Niederhauser (1999). 
5. Il est important de souligner, à ce propos, qu'en matière de domaines de spécialité, l'aliénation culturelle peut toucher toutes les classes, même les classes dites "supérieures", puisque le spécialiste se trouve "hors champ ", à part, à l'intérieur même de sa classe, enfermé dans son champ de spécialité.

6. Cette dimension perdure encore aujourd'hui pour les sciences dures.

7. Sur ce sujet, voir entre autres, Jacobi et Schiele (1988), A.-A. Moles et J.-M. Oulif (1967),

P. Maldidier et L. Boltanski (1977), B. Jurdant (2009), G. E. Ciapuscio (1993).

8. Dans ce cadre-là, il serait encore plus mal adapté de parler de médiation culturelle. Cet article ne peut traiter ici de ce sujet. Sur ce point, voir J. Caune (1999, 2000, 2006), B. Lamizet (2000).

9. Sur une définition des genres, voir C. Mast (2004) et C. Fasel (2008).

10. Voir E. Vargas (2005).

11. Ibid.

12. Dans notre nomenclature, les segments source sont signalés par des italiques, les segments reformulateurs par des caractères gras.

13. Voir E. Vargas (2005).

14. Nous distinguons ainsi «traduction interlinguale» et « code-switching (intralingual)». Sur le sujet, voir E. Vargas (2005).

15. La dénomination est ainsi souvent considérée comme le "contraire" d'une définition terminologique.

16. Sic.

17. Cet exemple renferme une double reformulation : une dénomination, puis dans un second temps, une reformulation définitoire.

18. La comparaison de brèves et de filets dans la presse française et allemandes montre bien que celles-ci ne sont souvent que des copiés-collés de communiqués de presse.

19. On peut évoquer ici la Reuters Trust Principles, une charte professant une volonté d'indépendance, d'impartialité et d'exactitude des faits rapportés, et de laquelle l'entreprise d'aujourd'hui se réclame encore.

20. Le terme est de nous.

21. Au sens d'O. Ducrot (1980 et 1984).

22. Voir E. Vargas (2009).

23. Communiqués et journaux spécialisés compris.

24. Au sens fourquetien du terme.

25. Sur l'anaphore, voir entre autres M. Charolles $(1991,2002)$ et G. Kleiber (1990 et 2001).

26. Contrairement à D. Jacobi $(1984,1988,1995)$, J.-M. Adam (1990), G. E. Ciapuscio (1993), ou les chercheurs réunis autour de S. Moirand et al. (1994) ou de P. Anderson et al., qui considèrent ces groupes comme autant de reformulations d'un premier élément cité, nous refusons de considérer ces segments anaphoriques comme "reformulations" à proprement parler: ces groupes nominaux renvoient certes au même référent, mais ils ne sont toutefois pas mis en relation les uns avec les autres comme le sont le dit et le redit unis par une opération de reformulation. Il n'y a pas entre les deux segments de retour réflexif du locuteur sur son dit premier.

27. Nous avons pu noter à ce sujet que les « toxische Wertpapiere » ou « faule Wertpapiere » sont massivement employés dans les quotidiens de gauche et fort absents des quotidiens dits conservateurs.

28. Nous remercions C. Resche pour ses informations sur le sujet. 


\section{RÉSUMÉS}

Cette contribution se propose d'analyser les discours de la presse allemande relatant la crise de 2008 lors de ses premiers signes visibles pour la société. Le cadre d'émergence du discours, la nature et les outils de celui-ci y sont analysés afin de dresser un portrait le plus complet possible du traitement des termes étrangers au lecteur profane ( mots de la crise »). C'est ainsi que cet article s'intéresse en particulier aux reformulations intratextuelles, aux chaînes anaphoriques et aux métaphores, posant par là-même la question de la vulgarisation et de la transmission de l'information dans la presse.

This paper aims to analyse the discourse of the German press in relation to the financial crisis of 2008 when its effects on society first began to appear. The context in which this discourse emerged, as well as its nature and linguistic construction, are studied in order to provide an accurate picture of how terms which would be unfamiliar for non-specialist readers were treated (i.e. the 'vocabulary of crisis'). The paper is concerned with intratextual reformulations, anaphoric linkages and metaphor, thus raising the issue of the vulgarization and transmission of current affairs in the press.

INDEX

Keywords : financial crisis, reformulation, vocabulary of crisis, vulgarization, written press Mots-clés : crise financière 2008, mots de la crise, presse, reformulation, vulgarisation

\section{AUTEURS}

\section{CLAIRE ALLIGNOL}

MCF, Université Stendhal - Grenoble 3. ILCEA

\section{ELODIE VARGAS}

MCF, Université Stendhal - Grenoble 3. ILCEA 